\title{
La gamificación como herramienta en el trabajo docente del orientador: innovación en asesoramiento vocacional desde la neurodidáctica
}

\author{
Sonia Clementina Ferrer Planchart; Mariana Fernández Reina; Noirailith Daniela \\ Polanco Padrón; Maria Eugenia Montero Montero; Estefany Elynett Caridad Ferrer*
}

Resumen. La innovación en el asesoramiento vocacional es un aspecto fundamental en el desarrollo de procesos que faciliten el trabajo docente del orientador educativo. Este documento tiene como propósito establecer algunas consideraciones sobre el uso de la gamificación como estrategia didáctica de la neurotecnología en el proceso de asesoramiento vocacional, con estudiantes de sexto grado de educación básica primaria. Metodológicamente contempla una revisión teórica sobre la Orientación como praxis científica, además de los fundamentos que explican la gamificación y el uso de las Tecnologías de la Información y Comunicación en el trabajo del Orientador. Se concluye que la utilización de videojuegos como práctica educativa fundamentada, con contenidos interesantes, fortalece el autoconocimiento de las potencialidades y el autoconcepto, además de proveer información sobre el mundo laboral, promoviendo ambientes de aprendizajes significativos que enriquecen las capacidades cerebrales de los estudiantes.

Palabras clave: gamificación; orientador; innovación; asesoramiento vocacional; neurodidactica; neurotecnología.

A GAMIFICAÇÃO COMO FERRAMENTA PARA O TRABALHO DOCENTE DO ORIENTADOR: INOVAÇÃO EM ORIENTAÇÃO VOCACIONAL NA NEURODIDÁTICA

Resumo. A inovação na orientação vocacional é um aspecto fundamental no desenvolvimento de processos que facilitem o trabalho docente do orientador educacional. O objetivo deste documento é estabelecer algumas considerações sobre o uso da gamificação como uma estratégia didática da neurotecnologia no processo de orientação vocacional com alunos do sexto ano do ensino fundamental. Metodologicamente, este artigo apresenta uma revisão teórica sobre a Orientação como práxis científica, além dos fundamentos que explicam a gamificação e o uso das Tecnologias da Informação e Comunicação no trabalho do Orientador. Conclui-se que o uso de videogames como prática educativa fundamentada, com conteúdos interessantes, fortalece o autoconhecimento do potencial e do autoconceito, além de fornecer informações sobre o mundo profissional, promovendo ambientes de aprendizados significativos que enriquecem as capacidades cerebrais dos estudantes.

Palavras-chave: gamificação; orientador; inovação; orientação vocacional; neurodidática, neurotecnologia

Universidad del Zulia (LUZ), Venezuela. 
THE GAMIFICATION AS A TOOL IN THE TEACHER'S WORK OF THE COUNSELOR: INNOVATION IN VOCATIONAL COUNSELING FROM THE NEURODIDACTIC Abstract. Innovation in vocational counseling is a fundamental aspect in the development of processes that facilitate the educational work of the educational counselor. The purpose of this document is to establish some considerations about the use of gamification as a didactic strategy for neurotechnology in the vocational counseling process, with sixth grade students in Primary Education. Methodologically, it contemplates a theoretical review on Guidance as a scientific practice, in addition to the foundations that explain gamification and the use of Information and Communication Technologies in the Guidance work. It is concluded that the use of video games as an educational practice based on interesting content, strengthens self-knowledge of potential and self-concept, in addition to providing information about the world of work, promoting significant learning environments that enrich the brain abilities of students.

Keywords: gamification; counselor; innovation; vocational advice; neurodidactic.

\section{INTRODUCCIÓN}

La complejidad del contexto socioeconómico, cultural y político de la realidad actual está impactando de forma directa a la sociedad. Esta transformación constante es una invitación a crecer asumiendo las crisis como oportunidades para el cambio y el bienestar común. En el caso de la educación las tendencias actuales se enfocan en modelos que resaltan la "construcción del conocimiento" tanto por los alumnos como por los profesores, ésta relación donde ambos tienen la capacidad de generar nuevos saberes contribuye con la adquisición de aprendizajes significativos. (Hernández y Hernández, 2004), especialmente con los descubrimientos realizados por la neurociencia sobre el funcionamiento del cerebro, lo cual supone un replanteamiento de las prácticas profesionales en distintas áreas del saber.

Esta perspectiva requiere de un docente diferente, el cual salga del enfoque tradicional, que fue funcional en su momento, para convertirse en un profesional creativo, autónomo, proactivo e innovador, que asuma retos, dando paso a nuevas realidades que incluyen construcciones sociales diferentes a partir del pluralismo que hoy presenta la posmodernidad. Por ello es necesaria una amplitud en las diferentes maneras de valorar y reflexionar sobre el impacto que tiene el docente y su práctica educativa en relación al desarrollo integral de sus estudiantes.

En este sentido se presenta el trabajo del profesional de la Orientación, como un docente comprometido con el fortalecimiento del potencial del ser humano en las diferentes áreas del conocimiento y en los contextos 
donde la persona se desarrolla. Al respecto, Vera (2011) presenta una visión holística de la orientación como disciplina proactiva, que está centrada en el proceso de asistir a las personas a aprender a utilizar sus recursos personales y ambientales para enfrentar las diferentes situaciones del vivir, que les permita desarrollarse de una manera saludable en su contexto social. Para Domínguez, Álvarez y López (2011) los profesionales de la Orientación educativa ocupan un lugar privilegiado por tener un rol de acompañamiento, ayuda y asesoramiento con la persona, por ello su importancia en la atención y desarrollo de procesos en todas las etapas de la vida.

En este marco conceptual, se plantea establecer en el presente documento algunas consideraciones sobre el uso de la gamificación como herramienta didáctica promovida por la neurotecnología, en el proceso de asesoramiento vocacional con estudiantes de sexto grado de educación básica primaria. Las razones que motivan este trabajo parten del interés por proporcionar a los estudiantes una estrategia lúdica que permita canalizar su autoconocimiento, intereses, gustos y habilidades en los primeros años de escolaridad, en contraposición a la aplicación de los test y otras estrategias estructuralistas, como recursos tradicionales en la exploración vocacional de los estudiantes, todo esto visto desde la perspectiva de la gamificación como propuesta metodológica educativa, basada en la neurotecnología.

Es pertinente explicar que en esta etapa de la vida se inicia la pubertad, en la cual, según Piaget, los cambios físicos comienzan a presentarse a la par de un pensamiento formal, además de que la persona desarrolla la capacidad de abstracción y de plantearse ciertas hipótesis por lo cual está en capacidad de asumir un programa o un proyecto de vida basado en la reflexión siguiendo un análisis hipotético - deductivo. (Sigüenza, 2018). Por ello, la importancia de brindar la orientación necesaria en el desarrollo de un proyecto de vida de estos estudiantes, quienes ya muestran cambios en su aspecto intelectual, asimilando y acomodando en su estructura cognoscitiva información externa de acuerdo a su nivel de desarrollo. Estas capacidades y el asesoramiento vocacional le permitirán afianzar las bases para su prosecución a la Educación Media General.

Al referirse al desarrollo vocacional del estudiante en esta etapa, Busot (1995) afirma que entre los 11 y 12 años de edad prevalecen los gustos y los intereses. Estas reacciones se dan a través del contacto con las asignaturas que le agradan o desagradan; a través de esas experiencias el estudiante logra diferenciar lo que le gusta, por resultarle placentero, de lo que le conviene (interés) conforme a su desempeño como estudiante. De igual manera, plantea el autor, que en esta edad todavía los intereses son inestables porque dependen de muchos factores, como su relación con sus 
padres en quienes confía, de quienes espera ayuda para tomar decisiones, aunque con el paso de su crecimiento va comprendiendo que la decisión final será suya, pues deberá elegir según criterios personales.

En este orden de ideas, diversos autores han investigado el tema de la vocación en la escuela primaria, fundamentalmente empleando la aplicación de test, charlas, talleres y programas entre otras estrategias (Quivera 2011, Fernández 2015). La mayoría de estos trabajos se ha enfocado en explorar la madurez o características vocacionales de los estudiantes de primaria y secundaria. Sin embargo, entre las investigaciones más recientes, no se han encontrado antecedentes sobre el uso de la gamificación para el desarrollo vocacional en primaria, por lo cual se considera que ésta sería una innovación en este campo de estudios. Por ello puede afirmarse que, abordar el desarrollo vocacional con esta nueva herramienta permitirá innovar en la praxis tradicional de la orientación vocacional, para incluir los juegos como una manera placentera de favorecer la socialización, las normas de convivencia, y, sobre todo, el objetivo principal de esta área disciplinar, favorecer el autoconocimiento mediante la exploración de los gustos e intereses de los estudiantes en esta etapa de la vida.

En este orden de ideas, Vygotsky (citado en Martín, 2017) considera el juego como una acción que orienta a la socialización y a los valores, además de ser un factor esencial en el desarrollo humano. En el mismo sentido, la UNESCO (1980) refiere que los juegos pueden proporcionar a la práctica pedagógica un medio para estimular la creatividad influyendo directamente en el desarrollo de la personalidad. En apoyo a estas posturas, Contreras (2016) expresa que los juegos permiten la experimentación de la identidad, la exploración de opciones y consecuencias, así como la prueba de los propios límites, ya que favorecen el desarrollo de las habilidades sociales, además de una diversidad de habilidades cognitivas propiciando el desarrollo del pensamiento lógico y crítico, aspectos claves para la resolución de problemas, la toma de decisiones y la planificación estratégica, entre otros procesos, todos los cuales son de vital importancia para el desarrollo vocacional de las personas. Al mismo tenor, Gross (citado en Martín, 2017) señala que el juego en la infancia es una preparación de las actividades que el individuo deberá realizar de mayor.

En este análisis se pretende brindar un aporte significativo para la innovación en asesoramiento vocacional con el uso de las tecnologías. Esta propuesta es un compromiso ético en el desempeño del Orientador asumiendo los cambios que el mundo globalizado presenta y que los docentes no pueden ignorar, sino asumir, entendiendo cada realidad y reto como oportunidades para construir proyectos socioeducativos en pro del bienestar de los estudiantes. 
Con base en estas exposiciones, se plantea entonces diseñar un prototipo de videojuego, basado en los postulados de la neurotecnología, específicamente desde la gamificación, para contribuir con el asesoramiento vocacional, el cual servirá como herramienta de trabajo del orientador en la ejecución de distintas estrategias para potenciar la formulación del proyecto de vida de los estudiantes, contribuyendo de esta manera su desarrollo vocacional.

\section{LA ORIENTACIÓN COMO DISCIPLINA CIENTÍFICA}

Es indudable que la orientación es una profesión enmarcada bajo preceptos teóricos que le dan sustento científico para su práctica, con estrategias que favorecen a los seres humanos en sus requerimientos personales, sociales, vocacionales, académicos y laborales, permitiéndoles elegir en una gama de posibilidades un estilo de vida de bienestar.

En este sentido, en el contexto educativo el orientador está llamado a desarrollar programas y servicios para abordar las diferentes situaciones que pudieran presentar las personas en todas las etapas de su vida. Estas circunstancias que se generan en la sociedad actual requieren de un ser humano capaz de enfrentar con éxito las diversas experiencias existenciales. Por ello, se hace necesaria la intervención a través de programas educativos que brinden las herramientas precisas para desarrollar habilidades para la vida obligatorias en el mundo actual. (Bisquerra y Álvarez, 2010).

Sin dudas, el trabajo docente del orientador en los centros educativos contempla desde la atención personal, social, académica, y familiar, hasta la puesta en marcha de programas para abordar requerimientos, como en este caso el área vocacional con niños de sexto grado en la escuela básica primari. Una de las razones que argumentan esta iniciativa, es la importancia de llevar a cabo acciones desde esta etapa del desarrollo para ir preparando a los estudiantes en su proyecto de vida, ya que usualmente tienden a esperar hasta cursar la Educación Media General para comenzar a tomar elecciones sobre alguna carrera profesional de su interés.

En este orden de ideas, Sobrado y Cortés (2009) plantean que la Orientación comprende una serie de funciones personales y grupales referidas a la información además del asesoramiento, de las capacidades e intereses para la toma de decisiones profesionales. En su praxis el orientador sustenta su trabajo con una diversidad de recursos materiales que facilitan el desarrollo de los procesos humanos, siendo hoy el uso de las tecnologías un importante medio para mediar conocimientos, proporcionando la enseñanza 
y el aprendizaje, además de la interacción entre los estudiantes y los docentes. Sobre es este tema, Domínguez, Álvarez y López (2011) plantean que las TIC facilitan el trabajo del orientador ya que son generadoras de nuevas vías de orientación y formación. Se entiende entonces que el uso de las tecnologías con recursos como el computador y demás dispositivos permite al orientador desarrollar actividades de formación que favorecen la creatividad y la producción del conocimiento.

\section{LA TECNOLOGíA COMO RECURSO QUE FAVORECE LA EDUCACIÓN}

Las Tecnologías de la Información y la Comunicación han desarrollado nuevas formas de relaciones sociales en el siglo XXI generando a su vez la llamada "Sociedad del Conocimiento". Estos recursos tecnológicos con que cuenta hoy el mundo han facilitado los avances en la salud, la educación, la ingeniería, la economía, y otros campos del saber en beneficio del hombre. En el contexto de la educación, la evolución que han alcanzado las TIC requiere de un sistema educacional actualizado en sus prácticas y contenidos, considerando el derecho que tienen las personas a recibir una formación acorde a la nueva sociedad de la información; por lo tanto, es necesario incorporar la tecnología al aula, lo que incluye una infraestructura tecnológica (hardware, software, y acceso a servicios de información y comunicación). Según UNESCO (2013) dentro de las políticas públicas del alcance de las TIC a favor de la educación, se plantean dos áreas de desarrollo prioritario para mejorar el sistema educativo: Nuevas prácticas educativas y medición de los aprendizajes.

De acuerdo a Riveros (2004) estos avances aportan suficientes elementos de análisis para dirigir desde el punto de vista técnico, un modelo actualizado del uso del computador en la escuela, siendo esta herramienta extremadamente útil en el proceso de enseñanza y aprendizaje de cualquier unidad curricular. Por su parte, Cabero (2017) plantea que la tecnología ha cambiado la manera de percibir la escuela ya que este importante recurso interviene en los medios de aprendizaje tanto del alumno como de los docentes que utilizan el internet como medio para investigar los contenidos a facilitar en las aulas de clase. Sin duda que las nuevas tecnologías ofrecen diferentes posibilidades para crear entornos de aprendizaje, poniendo a disposición de los estudiantes una amplitud de información con gran posibilidad de actualización. 
En este sentido, el papel de los docentes es cada vez más importante en los nuevos entornos educativos que se están creando y que se deben crear con la ayuda de las tecnologías de la información y la comunicación, por ello la innovación metodológica en la construcción de las actividades docentes es uno de los ejes fundamentales en los procesos de enseñanza y aprendizaje.

Por otra parte, Pradas (2017) afirma que el uso de la tecnología en la educación debe ser revisado también desde la neuropsicología, pues la comprensión de las bases sobre el cerebro, sus sistemas y su funcionamiento, puede aportar grandes ventajas al empleo de la tecnología en el aula con diversos propósitos. Esta autora manifiesta que el uso de la neurotecnología, concebida en sí misma como una metodología de aprendizaje que incluye el uso de los dispositivos y recursos tecnológicos disponibles para generar aprendizajes, es un campo de acción multidisciplinario enfocado más en el cómo aprender que en el qué aprender.

Esto es relevante si se consideran que, en el mundo moderno, la alta penetración que han tenido las tecnologías en la vida cotidiana de las personas, facilita la sobreabundancia de información en diversidad de formatos y en múltiples plataformas, desde las tradicionales como la radio y la televisión, pasando por las más actuales como la internet y las redes sociales; motivo por el cual se hace necesario que las personas, especialmente los estudiantes, sobre todo en los niveles básicos de la educación, desarrollen las habilidades de indagación, selección, análisis crítico, resolución de problemas, entre otras.

Por este motivo, Pradas (2017) asegura que, tomando en cuenta la evolución que han tenido las TIC en los últimos tiempos, la neurotecnología ha sido considerada como un método eficaz para lograr el éxito escolar de los estudiantes, no solo para proporcionar apoyos educativos a estudiantes con capacidades diferentes tales como dificultades de aprendizaje, trastornos de lenguaje, desarrollo del pensamiento, sino también para aprovechar las enormes capacidades tecnológicas que tienen los estudiantes, implementando actividades basadas en gamificación, fliped classroom, o conectivismo.

\section{ORIENTACION Y LAS NUEVAS TECNOLOGIAS DE LA INFORMACIÓN Y LA COMUNICACIÓN}

La Orientación como praxis científica, al igual que otras ciencias en la sociedad del conocimiento, comienza a dar sus pasos a principios de los años 90 en el uso de las nuevas tecnologías de la información y la comunicación, utilizando recursos que favorecen los procesos de información 
personal, académica y profesional como la toma de decisiones. Según Pantoja (2002, citado por Grañeras y Parras, 2009) en esta nueva tendencia en Orientación Educativa se plantean los siguientes temas a desarrollar con el uso de la tecnología: la auto orientación, el teletrabajo, la formación continua y adaptación a los nuevos puestos de trabajo, las nuevas formas de acceso al mundo del trabajo y las relaciones personales. Para este autor, desde ésta profesión se deben diseñar nuevos procesos de orientación y tutoría adaptados a la realidad de los centros educativos.

De modo que para lograr una utilización adecuada de los recursos que ofrecen las TIC en el proceso orientador, es necesario que los centros educativos mejoren la dotación tecnológica de los departamentos de orientación, así como es obligatorio que los docentes y orientadores adquieran una formación digital de calidad, solo así se podrán crear espacios virtuales en orientación de una manera fluida y flexible. (Campoy y Pantoja 2003). En este sentido Bisquerra (1996) señala que el modelo tecnológico en Orientación consiste en la utilización de los mass media (prensa, radio, televisión, videos, programas informáticos), con este modelo se puede atender simultáneamente a cantidades inmensas de personas. De igual forma, Pantoja (2004) refiere que el modelo tecnológico se basa en la integración de las nuevas tecnologías de la información y la comunicación en un programa de intervención dirigido a la prevención y al desarrollo, a tales efectos, el orientador es quien diseña y/o asesora en la relación que se produce entre la tecnología y el proceso orientador.

Se entiende entonces que la transformación de la Orientación con los aportes y el uso de las TIC invita a renovar el trabajo de los orientadores, pues estas innovaciones impactan la manera de intervenir cada proceso a abordar, por ello nuevas estrategias y recursos serían los medios para estar cerca de los diferentes escenarios sociales, económicos, culturales y políticos donde se desarrolla el ser humano.

\section{ORIENTACION VOCACIONAL}

La orientación vocacional dentro del sistema educativo está llamada a contribuir con la formación para la vida del sistema humano. No sólo implica la elección de una ocupación o profesión, es contribuir con la formación de un proyecto de vida. En este sentido la UNESCO (2001) señala lo siguiente

La orientación debería considerarse como un proceso continuo, que se extiende a lo largo de todo el sistema educativo, tener por objeto ayudar a todos a tomar decisiones conscientes y positivas en materia de educación 
y ocupación y velar porque se dé a los individuos los medios necesarios para: a) comprender exactamente cuáles son sus intereses, su capacidad y sus dotes especiales y estén en condiciones de tener un proyecto vital; b) cursar estudios o recibir una formación que les permitan desarrollar sus posibilidades y cumplir ese proyecto, c) adquirir la flexibilidad necesaria para adoptar decisiones sobre su ocupación, tanto en la fase inicial como en las ulteriores, que les encaminen a una carrera profesional satisfactoria, d) tomar en cuenta los factores económicos, sociales, tecnológicos, culturales y familiares que influyen en las actitudes y expectativas del educando y en la actividad profesional que elige...(p.19)

En apoyo a lo planteado, se considera que el orientador en ese proceso de asesoramiento fundamenta su trabajo apoyándose en diferentes enfoques. En el área vocacional desde su perspectiva desarrollista, se encuentra Super (1980), quien plantea que se trata de un proceso que tiene como objetivo ayudar a la persona a que elabore un concepto adecuado de sí mismo y de su desempeño en un trabajo. Este autor introdujo el concepto de madurez vocacional para señalar el grado de desarrollo individual desde el momento de sus primeras elecciones de fantasía en su niñez hasta sus elecciones en edades avanzadas. De igual forma, Busot (1995) refiere que cuando se piensa en la carrera profesional que se va a elegir, que se quiere ejercer en el futuro, se piensa en el logro de un proyecto de vida, por ello propone estrategias de orientación vocacional en el campo de la educación en todos los niveles. Culmina el autor afirmando que la conducta vocacional implica valores, intereses, aptitudes y gustos que se relacionan con alguna rama académica o alguna ocupación.

Otro aporte al tema vocacional lo presentan, Sobrado y Cortes (2009) quienes afirman que la Orientación como profesión tiene un rol importante en las decisiones que las personas tienen que tomar en las diferentes etapas de la vida, a su vez que potencia la elección profesional contribuyendo a la construcción de un proyecto de vida personal y profesional.

En Venezuela, la Orientación vocacional se ha desarrollado bajo los modelos de programas y servicios, siendo el modelo de servicios el más aplicado, bajo la figura de la administración de pruebas e instrumentos vocacionales que exploran intereses, aptitudes, gustos, y rasgos de personalidad, así como también actividades para dar información a los estudiantes sobre las ocupaciones, profesiones y contextos universitarios donde se ofrecen dichas formaciones. Uno de los instrumentos que lideriza la batería de pruebas en el país es la Prueba LUZ, cuya función esencial es orientar al estudiante de Educación Media General en su elección de carrera favoreciendo su ubicación en aquellas opciones profesionales de su preferencia, permitiendo a la vez presentar en los resultados los niveles de motivación y madurez vocacional. 
Actualmente, con el auge en la utilización de las TIC, la Prueba LUZ desde el año 2016 se transforma en la modalidad en línea, a través de una plataforma tecnológica que permite la accesibilidad desde cualquier lugar con conexión a internet, permitiendo también el ahorro de grandes recursos materiales y humanos que eran utilizados desde sus inicios para su aplicación, corrección y comunicación de resultados. En tal sentido las nuevas tecnologías, puestas al servicio de las actividades desarrolladas por el orientador ofrecen importantes alternativas para los procesos vocacionales, generando cambios e innovación en asesoramiento vocacional.

Atendiendo a estas consideraciones, la labor del orientador en la educación básica primaria, debe cambiar sus formas tradicionales de aplicación de test o pruebas exploratorias y abocarse a diferentes medios o metodologías activas que despierten en el estudiante el deseo de aprender y descubrir desde la experiencia que le aporta el juego y la compañía de otros sus capacidades, intereses, cualidades y aptitudes.

Por ello se presenta la gamificación como herramienta tecnológica innovadora que aporta desde el juego encuentros vivenciales para despertar las emociones y la motivación por aprender desde una manera diferente de abordar la orientación vocacional.

\section{GAMIFICACIÓN COMO HERRAMIENTA EN ASESORAMIENTO VOCACIONAL}

Teniendo en cuenta que el proceso educativo actual enfatiza al aprendiz como motor de acciones derivadas de la formación que representan un papel protagónico en la dinámica social, cultural y económica en tiempos modernos; resulta oportuno emprender el análisis de alternativas para planificar recursos novedosos dirigidos a estudiantes del presente siglo, que vienen cargados de tecnología y revelan la necesidad de maximizar las fuentes de contribución al desarrollo individual, entre los que se destacan los elementos de gamificación en el currículo integral.

El concepto de gamificación tiene origen anglosajón y fue introducido en el año 2008, y comenzó a utilizarse en el mundo de los negocios como estrategia de mercadeo, en la cual se aplicaban elementos del juego para atraer y persuadir a los usuarios en la realización de una acción. Este procedimiento puede extrapolarse a cualquier ámbito social, entre ellos el educativo, en el cual esta estrategia ha ganado auge. (Observatorio de Innovación Educativa del Tecnológico de Monterrey, 2016). 
Cuando se define el término de gamificación o ludificación, se hace referencia a la adopción de mecánicas, instrucciones y componentes particulares de los juegos, aplicable en cualquier contexto organizacional. Según Kapp (citado en Pradas, 2017) este plan incluye precisar la motivación de acciones o comportamientos requeridos para promover métodos efectivos para estudiar - aprender, o en líneas generales poder resolver problemas situacionales.

Por su parte, Sánchez i Peris (2015) señala que la gamificación puede ser concebida como técnica y estrategia paralelamente, partiendo de la noción de involucrar múltiples naturalezas que resultan atractivas en las dinámicas lúdicas y que repercuten favorablemente en la vinculación de los estudiantes con el medio de aprendizaje, incentivo a modificaciones de comportamiento diario o hacer difusión a un determinado mensaje o contenido con el cual se pueda crear una experiencia motivadora para el participante.

Entre los beneficios de la gamificación en el aula, la Pontificia Universidad Católica de Valparaíso (PUCV) (2017) señala los siguientes: a) genera retroalimentación oportuna a los estudiantes; b) proporciona información al docente del curso; c) fomenta la relación entre pares y equipos; d) promueve instancias de aprendizaje activo; e) mejora los aprendizajes de los estudiantes; y f) motiva a los estudiantes a participar activamente en la clase.

Como visión general, los métodos propuestos en gamificación plantean ciertos procesos no-lúdicos como transitorios hacia los juegos, donde el sujeto asume el rol de jugador y personifica el eje central del juego, resultando determinante administrar acciones relacionadas a la toma de decisiones propias, sentido de progreso ante retos encontrados, participación efectiva en espacios de interacción social, dotarse de reconocimientos originados por los logros adquiridos, inmediatez en la retroalimentación con grupos de interés común, así como experiencias inmersivas y divertidas dentro de entornos dinámicos propuestos para el fortalecimiento de competencias.

En el ámbito de las ciencias de la educación, de manera tácita se asocia a la gamificación con la incorporación de atributos de diseño de juegos para aprovecharlos en los encuentros de instrucción, siendo indudable, además, que la planificación de una clase gamificada pudiese ser sostenida por el docente orientador en la dinamización del seguimiento académico-personalprofesional de sus estudiantes. La gamificación puede ser considerada como estrategia didáctica y motivacional adoptable en el proceso educativo, de utilidad para provocar comportamientos específicos en el estudiante dentro de un ambiente creativo y atractivo, que genere un compromiso con la actividad en la que participa y que apoye al lucro de experiencias positivas de aprendizaje. No se trata de utilizar juegos en sí mismos, sino de tomar parte 
de las mecánicas, medios de incentivos, narrativa, retroalimentación entre usuarios, reconocimientos, alternativas de progresión entre niveles, sistemas de puntaje, entre otros elementos característicos que logren enriquecer las rutinas de aprendizaje. (Ortiz-Colón, Jordán y Agreda, 2018).

Siguiendo este orden, lo señalado por Borrás (2015) resume que los elementos del juego se entienden como piezas útiles para gamificar actividades del aula, partiendo de las mecánicas como estrategias de creación de dinámicas lúdicas que incluyen sistemas de connotación y demostración de progreso, entre los que se pueden mencionar los avatares, puntuación, emblemas de refuerzo positivo, clasificaciones, retos, componentes estéticos, colores, medallas, interfaz gráfica. Del mismo modo, la historia de la experiencia de juego, como un punto primordial para activar expectativas en el jugador y lograr su identificación con el mismo.

Adicionalmente, los juegos permiten crear situaciones de experimentación práctica para desarrollar la inteligencia emocional y social, al ser intrínsecamente motivadores, siendo destacable que no se trata de "jugar por jugar", la dinámica lúdica debe marcar sus orientaciones hacia los resultados de formación perseguidos, promoviendo instancias de aprendizaje activo, generando retroalimentación entre participantes, planificando la clase en función del contenido, selección del juego, sistema de recompensas, reglas y tiempo a utilizar; conjuntamente con la retroalimentación de resultados (PCUV, 2017).

En concordancia con las ideas expuestas, el trabajo del docente orientador puede utilizar elementos de juego para alentar a los grupos a que se sumerjan en sus intereses, en las necesidades de su ambiente y en la concepción de su mentalidad de crecimiento, lo cual, emana desde los principios de la neurodidáctica. La gamificación de la praxis del docente orientador lograría encaminar la motivación de acciones para aprender y socializar con emoción, partiendo del juego como influencia directa en los mecanismos básicos para desarrollar el cerebro emocional y cognitivo a la vez. Gamificar las prácticas de asesoramiento vocacional permitirá al orientado tener activismo en su participación ya que el cerebro es social, pudiendo influenciar positivamente los comportamientos cooperativos, mejorar habilidades, proveer aproximaciones innovadoras en los procesos de evaluación, fomentar competencias amigables entre usuarios y, por supuesto, rediseñar los estándares para la adquisición de saberes sobre aquello que conoce y podría llegar a dominar.

El alto potencial que posee la gamificación para moldear el comportamiento de los usuarios es atrayente para ser adaptado a su contexto situacional, y directamente puede ser aplicado por el docente como herramienta de apoyo en la propia exploración personal de un estudiante consigo mismo; ya 
que en efecto, la adopción de mecánicas lúdicas basadas en entretenimiento digital, puede proporcionar valiosos recursos que brinden soporte al estudiante a seguir procesos particulares, usar guías para el autoconocimiento, tomar decisiones vocacionales, mejorar su autonomía y continuar sus experiencias de aprendizaje de manera diferente y divertida.

En función con lo referenciado, es claro que la postura de docentes orientadores y académicos se entiende hoy, como trabajo para la transformación de las comunidades, incluyendo habilidades que anteriormente quizás no compaginaban con la realidad situacional o las necesidades educativas del momento. Aunado a esto, la organización de aulas con implantación de tecnologías ha subrayado flamantes preferencias para el presente siglo, donde el marco educativo queda igualmente centrado en la formación inclusiva y adjunta a diversos ejes transversales, junto con la participación de cada miembro integrador del sistema hacia la potenciación de competencias, resultando así, que los nacientes movimientos conjeturan márgenes firmes para el desarrollo independiente de estudiantes, quienes al verse moderados por instrumentos de interacción digital, podrían alcanzar el empoderamiento de las plataformas dispuestas para emplear y llegar a ser más diestros en su plano personal o profesional.

Así, sucesivamente las orientaciones en las aulas deberán ser más participativas, basadas en la adecuación de un currículo que adicione estrategias para el desarrollo de competencias específicas, en lo conceptual, procedimental y, con énfasis, en lo actitudinal; el potencial cerebral de los infantes es enorme, por tanto, es tarea del docente inclinar sus esfuerzos hacia la aplicación de aprendizajes cooperativos, ejecución de proyectos y autoconocimiento del ser, porque es un hecho que, al incrementar los niveles de atención en determinados casos, generar confort en clases, crear hábitos de estudio, mantener un ritmo de aprendizaje entretenido y dinámico, hasta utilizar metodologías activas para la exploración cerebral, entonces los educandos serán capaces de complementar, reflexionar, criticar posturas, así como aplicar y negociar giros en la estructura propuesta en el currículo escolar.

\subsection{Diseño conceptual del prototipo}

Considerando la exposición anterior, se pretende conceptualizar, diseñar y desarrollar un videojuego que pueda servir como pasarela de apoyo al trabajo docente del orientador para ser aplicado con estudiantes de sexto grado de educación básica primaria en la exploración de su propio perfil vocacional; con el objetivo de implementar encuentros de orientación personal basados en técnicas de gamificación, a través del manejo de un software 
hecho a la medida. La principal motivación que ha propiciado la realización de una propuesta para la confección de una plataforma digital de asesoramiento vocacional en niños de edad escolar ha sido, especialmente, el interés en trabajar en el campo del autoconocimiento. Al mismo tiempo, el campo de las ciencias informáticas extiende oportunidades para la integración de labores técnicas, aplicación de metodologías ágiles, diseño y programación de sistemas específicos, mejoría de habilidades de psicomotricidad dadas por juegos tipo plataformas y, cardinalmente, dotación de oportunidades para recrear y desatar diversos sentimientos mediante experiencias simuladas digitalmente por parte de los usuarios finales, a la vez que aprenden de sí mismos, se divierten y comparten en su entorno colectivo.

\subsection{Diferentes roles del equipo de desarrollo}

El desarrollo de la propuesta de software será una tarea llevada a cabo por un equipo multidisciplinar donde convergerán diversos perfiles ocupacionales de campos variados en ciencias. Los roles que se necesitarán varían, según la naturaleza de un determinado proyecto, sin embargo, sobre esta base, cabe destacar que para una aplicación ideada hacia sistemas móviles, no resulta apremiante un equipo numeroso de integrantes para afrontar todos los roles del proyecto, por tanto, se considerarán para las labores los tres (03) roles globales de todo proceso de producción, a ser ocupados por un grupo que quedará sometido a captación y formación posterior, a saber: especialista (orientador experto en labores de asesoría vocacional, encargado de conceptualizar todo el contenido base para el sistema), programador (personal encargado de crear y escribir códigos para dar funcionalidad a lo diseñado) y diseñador (quién construiría las mecánicas, reglas e interacciones para el jugador, respuestas visuales y sonoras, guión y niveles, asimismo, deberá ser agente de transmisiones eficaces sobre lo que se quiere de cada miembro del equipo, plasmaría requisitos y evitará rehacer el trabajo).

\subsection{Visión general del sistema: plataforma de gamificación} para el asesoramiento vocacional de estudiantes de sexto grado

- Género: exploración, educativo.

- Plataforma destino: Windows/Linux.

- Clasificación: E - Everyone: apto para todo público, puede contener caricaturas, fantasía / E 10+ Everyone +10 (para todos a partir de diez años). 
- Mecánica del juego: desplazamiento entre mundos, manejo de respuestas, cuestionarios, misiones a cumplir desde los intereses y gustos.

- Público al que se dirige: niños de sexto grado de educación básica.

- Estilo de arte e ilustraciones del juego: caricaturas y modelos 3D.

- Concepto: se busca desarrollar un videojuego que reúna una serie de ítems específicos sobre exploración personal, donde el jugador se visualice dentro de distintos entornos que recreen diferentes partes del mundo. Cada jugador, al iniciar el sistema, deberá personalizar su propio avatar, para crearse a sí mismo dentro del juego. Se iniciará identificando la partida con los datos personales de cada usuario y se podrá activar una primera pantalla donde puedan dar una breve descripción de sí mismos, usando por supuesto palabras propias. Los jugadores podrán a su vez, encontrarse con personajes conocidos en función del mundo donde transiten y su propio personaje podrá contar progresivamente con ciertas habilidades que incluirían poderes de transformación, para apuntar a la fantasía que tanto envuelve y motiva a la juventud.

La perspectiva inicial, procurará que el aspecto gráfico y narrativo del videojuego se cuente a modo de viaje por el mundo. La jugabilidad estará basada en el tipo de juegos de selección múltiple, donde el estudiante podrá hacer elecciones de datos, emblemas, colores, y preferencias varias, que tendrán un trasfondo en su actividad de orientación. Entre los ítems que se pretenden abordar dentro de la jugabilidad de la plataforma de gamificación, se incluyen juegos sociales (duales o multijugadores), competencias sencillas, creación de historias de sí mismos, elección de opciones, juego de memoria, reconocimiento de patrones, dinámica familiar, entre otras estrategias que permitan favorecer el entrenamiento cerebral, ofrecer experiencias al usuario altamente emocionantes y brindar indicadores efectivos para la generación de resultados del trabajo del orientador. Este juego contaría con un sistema de múltiples niveles de ascenso, que el usuario jugador deberá recorrer, enmarcado en diferentes espacios y fusionando ambientes reales y ficticios. Estos niveles tendrán un inicio y fin, con la misión de recuperar alguna clase de insignia que estaría presente en cada nivel, teniendo que, en cada uno de ellos deberá superar una misión final que accionaría la apertura a la siguiente actividad. 


\section{REFLEXIONES FINALES}

Es un hecho conocido que las TIC han revolucionado el modo de vida de las personas en las últimas décadas. Puede verse cómo una gran cantidad de dispositivos electrónicos y digitales se emplean a diario para solucionar los problemas encontrados y facilitar los procesos de la vida cotidiana. Es así como la tecnología se ha usado ampliamente en el campo educativo, pues, como lo han demostrado innumerables estudios, ésta puede propiciar un aprendizaje significativo y perdurable en el tiempo, aprovechando las habilidades y competencias que los estudiantes han desarrollado para hacer frente a su aprendizaje en un mundo digital, y propiciando un ambiente de aprendizaje motivador

Este hecho ha generado una modificación en la práctica educativa de los orientadores, profesionales dedicados a potenciar el desarrollo humano en distintas áreas, siendo una de estas la orientación vocacional, en la cual la persona desarrolla un proyecto de vida, basado en sus gustos, intereses, valores, expectativas y experiencias, que se irá cristalizando y consolidando en la medida en que se produce su desarrollo humano.

En este sentido, las nuevas tendencias educativas, entre ellas la neurodidáctica, ofrece algunas posibilidades para innovar en el trabajo del orientador en el área vocacional, entre ellas la gamificación, mediante la cual se busca lograr la participación de la totalidad del cerebro en el desarrollo de experiencias lúdicas diseñadas cuidadosamente con distintos fines.

Un área en la cual convergen la orientación y la neurodidáctica mediante la gamificación, es la orientación vocacional en estudiantes de sexto grado de Educación Primaria, ya que, mediante un videojuego enfocado en la prática de roles y la simulación de experiencias laborales y ocupacionales, puede aportar al desarrollo del proyecto de vida de los jóvenes estudiantes, propiciando además el fortalecimiento de sus competencias tecnológicas e informacionales, tan preciadas en la vida cotidiana del mundo moderno.

La creación, implementación y evaluación del prototipo de este videojuego para el desarrollo vocacional de estudiantes de sexto grado, constituye una contribución desde la orientación como disciplina científica al incremento de datos e informaciones relevantes en el área de la neurotecnología educativa, a la vez que propicia el aprovechamiento real del potencial que poseen las aplicaciones y software educativo para el aprendizaje, dejando de ser simples recursos de apoyo para presentar información, los cuales además generan una mayor motivación para el aprendizaje, aprovechando además las capacidades tecnológicas de esta generación de jóvenes adolescentes. 


\section{BIBLIOGRAFÍA}

Borrás, O. (2015). Fundamentos de la gamificación. Universidad Politécnica de Madrid. Gabinete de Tele-educación. Recuperado de: http://oa.upm.es/35517/1/fundamentos\%20 de\%20la\%20gamificacion_v1_1.pdf

Busot, A. (1995). Elección y desarrollo vocacional. Maracaibo: Ediluz.

Cabero, J. (2017). La formación en la era digital: ambientes enriquecidos por la tecnología. Revista Gestión de la innovación en educación superior. Vol. 2(2), pp. 41-64. Recuperado de: http://ojs.inacap.cl/index.php/regies/article/view/24

Campoy, T. y Pantoja, A. (2003). Propuestas de e-orientación para una educación intercultural. Comunicar, 20, 37-43. Recuperado de: http://www.quadernsdigitals.net/datos/ hemeroteca/r_2/nr_665/a_8946/8946.pdf

Cárdenas N. (2005). Ética, actitudes y habilidades socioemocionales en la relación pedagógica. Valencia Venezuela: Impresos rápidos.

Deterding S., Dixon, D., Khaled, R. y Nacke, L. (2011). From game design elements to gamefulness: defining gamification. 15th International Academic MindTrek Conference: Envisioning Future Media Environments. 9-15. doi>10.1145/2181037.2181040

Domínguez, G., Álvarez, F. y López, E. (2011). Orientación Educativay Tecnologías de la Información y la Comunicación. Nuevas respuestas para nuevas realidades. Sevilla: MAD.

Fernández, A. (2015). Tutoría y desarrollo vocacional en la educación primaria. Trabajo de fin de grado. Universidad Internacional de la Rioja, España.

Grañeras, M. y Parras, A. (Coord.) (2009). Orientación educativa: fundamentos teóricos, modelos institucionales ynuevas perspectivas. (2da. edición). España: Ministerio de Educación.

Hernández, A. y Hernández, A. (2004). Características de la enseñanza constructivista en docentes de Matemática. Omnia año 10, n³ 105-126.

Kapp, K. (2012). The Gamification of Learning and Instruction: Game-based Methods and Strategies for Training and Education. San Francisco, California: Pfeiffer.

Martín, J. (2017). Gamificación en el aula de ELE. (Trabajo de Fin de Grado en Español: Lengua y Literatura). Universidad de Valladolid, España.

Moll, S. (05/06/2014). Gamificación: 7 claves para entender qué es y cómo funciona (documento en línea). Recuperado de: http://justificaturespuesta.com/gamificacion-7-clavespara-entender-que-es-y-como-funciona

Observatorio de Innovación Educativa del Tecnológico de Monterrey. (2016). Gamificación. Reporte Edutrends. Recuperado de: http://eduteka.icesi.edu.co/pdfdir/edutrendsgamificacion.pdf

Ortíz-Colon, A., Jordán, J. y Agreda, M. (2018). Gamificación en educación: una panorámica sobre el estado de la cuestión. Educação E Pesquisa, 44, pp. 1-17. doi: http:// dx.doi.org/10.1590/S1678-4634201844173773

Pontificia Universidad Católica de Valparaíso (PUCV) (2017). La gamificación en el proceso de enseñanza y aprendizaje. Unidad de Mejoramiento de la Docencia Universitaria (PUCV). Recuperado de: http://vra.ucv.cl/ddcyf/wp-content/uploads/2017/03/ gamificacion_continua.pdf 
Pradas, S. (2017). Neurotecnología educativa. La tecnología al servicio del alumno y del profesor. España: Ministerio de Educación, Cultura y Deporte. Secretaría de Estado de Educación, Formación Profesional y Universidades. Centro Nacional de Innovación e Investigación Educativa. Disponible en: https://cazadetalles.files.wordpress. com/2017/01/libro-de-neurotecnologia-unir.pdf

Quivera, I. (2011). Madurez vocacional y características personales en estudiantes de educación básica primaria. (Trabajo especial de grado para optar al título de Magister en orientación). Universidad del Zulia, Maracaibo.

Riveros, V. (2004). Las Tecnologías de la Información y la Comunicación y el proceso de enseñanza y aprendizaje de la matemática: algunas consideraciones. Omnia 10(3). Recuperado de: http://produccioncientificaluz.org/index.php/omnia/article/view/7114/7103

Sánchez i Peris, F. (2015). Gamificación. Education in the Knowledge Society, vol. 16 (2). pp. 13-15. Universidad de Salamanca. España. Recuperado de: http://www.redalyc.org/ pdf/5355/535554758002.pdf

Sigüenza, J. (2018). Piaget y la educación obligatoria en México. RIEL, Año 2 (2), pp. 73-80. Centro Regional de Formación Docente e Investigación Educativa. Recuperado de: http://cresur.edu.mx/OJS/index.php/RIEL_CRESUR/article/view/149/128

Sobrado, L. y Cortés, A. (2009). Orientación profesional: Nuevos escenarios y perspectivas. Madrid: Biblioteca nueva.

Stortoni, M. (2017). Tríada didáctica y legitimación de autoridad: dos factores necesarios para generar conocimiento. Escritos en la Facultad, Año 13(136), pp.35-36. Universidad de Palermo, Argentina. Recuperado de: http://fido.palermo.edu/servicios_dyc/ publicacionesdc/archivos/684_libro.pdf\#page=36

Super, D. (1980). Psicología de los intereses y las vocaciones. Buenos Aires: Kapelusz.

UNESCO (1998) El niño y el juego. Planteamientos teóricos y aplicaciones pedagógicas N³4 Estudios y documentos de Educación. Recuperado de: http://unesdoc.unesco.org/ images/0013/001340/134047so.pdf

UNESCO (2001). Conferenciageneral. Recomendación relativa a la enseñanza técnica. Recuperado de: http://portal.unesco.org/es/ev.php-URL ID=13145\&URL DO=DO TOPIC\&URL SECTION=201.html

UNESCO (2013) Enfoques estratégicos sobre las TICS en educación en América Latina y el Caribe. Recuperado de: http://www.unesco.org/new/fileadmin/MULTIMEDIA/FIELD/ Santiago/images/ticsesp.pdf

Vera, G. (2000). Pedagogía y formación de Orientadores: Una perspectiva constructivista. Revista Pedagógica. Volumen 24 (69). Caracas.

Vera, G. (2011). La Orientación como política de estado y el sistema nacional de Orientación. Un avance educativo en prevención, salud mental y progreso socio-económico venezolano. Recuperado de http://servidoropsu.tach.ula.ve/7jornadas_i_h/paginas/ doc//JIHE-2011 PT019.pdf 